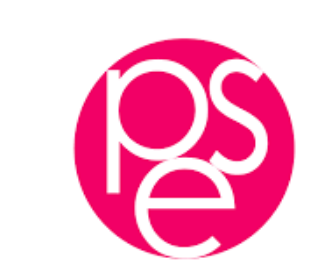

PARISSCHOOL OFECONOMICS
ECOLED'ECONOMIEDE PARIS

WORKING PAPER N ${ }^{\circ} 2018-16$

Decision Under Psychological Pressure: The Shooter's Anxiety at the Penalty Kick

\author{
Luc Arrondel \\ Richard Duhautois \\ Jean-François Laslier
}

JEL Codes:

Keywords:

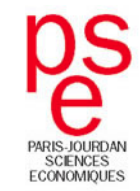




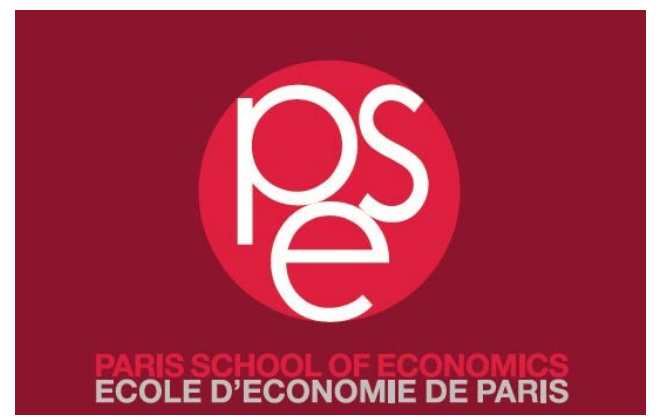

\title{
Decision Under Psychological Pressure: The Shooter's Anxiety at the Penalty Kick
}

\author{
Luc Arrondel \\ CNRS-Paris School of Economics-FCN \\ 48 Boulevard Jourdan \\ 75014 Paris, France \\ arrondel@pse.ens.fr \\ Richard Duhautois \\ CNAM-Lirsa and Ceet \\ richard.duhautois@lecnam.net \\ Jean-François Laslier \\ CNRS-Paris School of Economics-SdR \\ 48 Boulevard Jourdan \\ 75014 Paris, France \\ jean-francois.laslier@ens.fr
}

April 2018

Acknowledgement: 


\section{Decision Under Psychological Pressure: The Shooter's Anxiety at the Penalty Kick}

Abstract:

The paper studies sequences of penalty kicks during football shoutouts in French cup competitions. We seek to analyze in detail the psychological effects to which the kicker responds: fear of winning, fear of losing, expected outcomes or how much is at stake. The main conclusion of our study is that the performance (the probability of scoring) is impacted negatively by both what is at stake (the impact of my scoring on the expected probability that my team eventually wins) and by the difficulty of the situation (the ex ante probability of my team eventually losing). We find no advantage for a team to take the first kick.

"You know what? The truth is, I've just taken the penalty kick." Eric (The King) Cantona, 1994

\section{Introduction}

Behavioural economics has challenged the "standard" rationality of homo economicus, in particular in view of the cognitive biases, if not "emotions", affecting behaviour. This criticism has in effect introduced other psychological parameters than the most classic ones, to explain individual decisions. For example, in savings, individuals' risk aversion and time preference are no longer the only factors raised to explain individuals' investments. Also considered today are individuals' loss and ambiguity aversion and optimism/pessimism, if not feeling of "fear", especially during crises (Guiso and Sodini, 2013).

Decision-making has been shown to be influenced by physical and psychological factors such as hunger (Ashton et al., 2017), stress (Porcelli and Delgado, 2009), sexual arousal (Ariely, 2008) and other "emotions" such as fear, anger and happiness (Nguyen and Nousssair, 2014). Such studies demonstrate that these somatic and psychological conditions partially determine which decision is made by the decision-maker by altering, for example, his or her attitude to risk. 
This article looks into the role of psychological pressure on individual decision-making in a competitive environment. In the case of investment behaviour, this concerns, for example, the on-the-spot decisions that traders have to make on the trading floor. Yet our terrain is different, as we focus on football and the penalty shoot-outs that determine the outcome of a match when both teams are tied at the end of the regulation playing time (and extra time). In other words, our study examines the capacity to pull off a relatively simple move (for trained footballers at least) in a competitive environment.

Compared with the abovementioned studies, the case of the penalty kick differs because the "decision" here is a sport move. The sport penalty kick is both more straightforward and more complicated than an economic-type decision. It is more straightforward because, once the shooter has decided where to kick (to the right, left or centre, high or low, etc.), there are no more subtle questions of strategy, uncertainty or weighting between cost and expected return: it is quite simply a matter of performing the planned move as best as I can. Yet the sport move is also more complicated because it involves complex cognitive, nervous and muscular mechanics for its execution. When a trader decides on a selling price, it is not a problem to execute the decision. Yet the penalty kick is a good example of virtually instant individual execution with different quality outcomes.

A number of economists have already looked into this sport exercise to analyse the role of "psychological pressure" on its success, especially during penalty shoot-outs designed to determine the outcome of a match (draw). They have focused, in particular, on whether there is an advantage to being the first team up in the shoot-out, i.e. taking the first kick. This question has sparked a controversy. On one side, Apesteguia and Palacio-Huerta (2010) find that the team that takes the first kick wins the penalty shoot-out $60 \%$ of the time. On the other side, Kocher et al. (2012) find no advantage from their larger sample to being the team to take the first kick. The former finds psychological pressure on the second kicker, whereas the latter find no such pressure. As ours is a study of scoring or missing a penalty kick during a shootout, we also have our say in this controversy.

The abovementioned studies analyse a number of penalty shoot-outs during the following tournaments: World Cup, European Championship, Copa America, and Spanish, English and German cups. We propose here an analysis of penalty shoot-outs in French cup competitions: the League Cup ("Coupe de la Ligue"), the football association (FA) Cup ("Coupe de France") and the Champions Trophy (Le tropheé des champions"). 
Our results find no advantage to taking the first kick: both teams have equal chances of winning if they start the shoot-out or if they kick second (other things being equal). The probability of scoring one's penalty kick therefore does not depend on being first up. Note that the starting team is randomly determined (a coin tossed by the referee), which makes all penalty shoot-outs a particularly pure example of a randomized natural experiment. It might therefore seem surprising to find a controversy raging over such a straightforward statistic as the frequency of winning.

Yet, aside from this controversy of interest to football fans, the penalty kick forms a remarkable case study for the analysis of individual actions "under pressure". The penalty kick is a relatively complex, but strictly individual and often repeated motor action. Its outcome is perfectly defined (the goal is scored or not) and partially random (it therefore lends itself well to statistical analysis). Apesteguia and Palacio-Huerta (2010) argue that the existence of an advantage to taking the first kick proves the existence of a psychological effect on the shooter, even professional, whose capacity to score decreases when his team is lagging behind. We will see in this paper that different psychological effects can indeed be found, even though they secure no advantage for the team taking the first kick.

The work presented here seeks to analyze in detail the psychological effects to which the kicker responds: fear of winning, fear of losing, expected outcomes or how much is at stake. We therefore consider other hypotheses that might explain why shooters score or miss their penalty kicks: difference in score for the same number of penalty kicks, the impact of the "stake", relative "importance" of the penalty kick, etc. The main conclusion of our study is that the outcome of a penalty shoot-out depends on many other psychological factors than just the "pressure" of shooting after an opposing team player...

The article is structured as follows. Section 2 is a review of the (abundant) body of literature on the subject. Section 3 presents some factual elements on the rules that govern penalty kicks, the data used in this study and a few basic descriptive statistics. Section 4 develops our analysis, with (4.1) based on strictly football considerations (new or already discussed), and (4.2) building new variables in the literature on penalties, but this time directly derived from the usual concepts of decision theory under risk. Section 5 concludes. 


\section{The science of the penalty kick and other sport moves}

The impact of pressure on performance has been studied in many sports, form weightlifting (Genakos and Pagliero 2012) to golf (Hickman and Metz 2015) and chess (Gonzales-Diaz and Palacios-Huerta 2016). This literature is reviewed in Bellok and Gray (2007) and Hill et al. (2013). In football, the penalty kick has existed for 126 years. It was introduced into the Official Laws of the Game by the International Football Association Board (IFAB) in 1891 at the initiative of Irish goalkeeper for Milford Everton FC, William McCrum. Since 1970, all major football tournaments - international events and national competitions - have held penalty shoot-outs to replace the coin toss when a match ends in a draw. The outcome of these penalty kicks depends on a number of factors such as skill and fatigue, but also obviously on psychological aspects.

\subsection{How to score a penalty}

Numerous studies have studied the strategic interaction between the kicker and the goalkeeper who are playing a kind of hide-and-seek game (each would win if he knew what the other is going to do) and have concluded that professional players master this game remarkably well (Chiappori et al. 2002; Bar-Eli et al. 2007; Botwell et al. 2009; Azar and Bar-Eli 2011, among others). But this strategic optimality in the choice of strategies is by no mean exclusive of non-rational psychological factors that might impact the quality of the kick.

\begin{tabular}{lccc}
\hline & Table 1: Penalty kicks in Football between 2006/07 and 2015/16 \\
\hline League & Number of penalties & Number of penalties scored & Proportion of penalties scored \\
Premier League & 948 & 739 & 0.7795 \\
Bundesliga & 745 & 571 & 0.7664 \\
Liga & 1089 & 831 & 0.7631 \\
Serie A & 1239 & 945 & 0.7627 \\
Ligue 1 & 931 & 708 & 0.7605 \\
\hline
\end{tabular}

Sources : National Leagues

Chiappori et al. (2002) find that $90 \%$ of penalty kicks are scored during training sessions as opposed to $80 \%$ during actual matches, suggesting that psychological aspects are at work. The percentage of penalties scored in the main League championship is actually slightly lower, at around $77 \%$ (Table 1). And this percentage is even lower in shoot-outs than in matches.

Psychology can take different forms for players who take penalty shoot-outs: being the first or last kicker (Jordet et al. 2007; McGarry and Franks 2000); dealing with anxiety between the end of extra time and the penalty kicks (Jordet and Elferink-Gemser 2012); playing on the last team to score before the penalty shoot-out (Lyttleton, 2016); playing on a particular team or 
being or not being a star (Jordet, 2009); coming from a particular country (Billsberry et al. 2007); and wearing a red shirt (Greenlees et al. 2008).

Jordet et al. (2006) undertake to estimate the relative importance of different factors in international penalty shoot-outs. They use data from World Cups, European Championships (Euro) and South American Championships (Copa America) from 1976 to 2004 (41 shootouts). They show that psychological factors account more than other factors for the outcome of penalty kicks. Specifically, kick order number appears to be decisive in that fewer goals are scored at the end of shoot-outs when it becomes vital to score. However, it is hard to disentangle psychology and skill, because the more highly skilled players often take the penalty kicks first, influencing the probability of scoring. Although the authors do stress this fact, it is not easy to identify the two factors. Interestingly, with respect to fatigue, they find no significant relationship between playing time and kick outcomes. Lastly, their results suggest that stress and anxiety may be important explanatory factors.

Studying the performance of professional players in penalty kick situations the 3,500 penalty kicks in 40 years at the German Premier football league), Dohmen (2008) finds a strong detrimental effect of the presence of supportive (home) spectators but no effect of the importance of the kick.

Jordet and Elferink-Gemser (2012) observe the dynamic process of stress and anxiety based on their interviews with elite professional soccer players who played in the match between Sweden and the Netherlands in one of the 2004 European Championship quarter finals in Portugal. They decompose the penalty shoot-outs into four phases: the break after extra time, the mid-circle (before the kick), the walk (to kick) and at the penalty mark. One of their interesting results is the dynamic process of anxiety: it "tends to reach a peak in the midcircle, and then progressively decrease as the shooter starts walking towards the penalty mark."

Anxiety can vary depending on different events that take place during the game. When you get to the point of a penalty shoot-out, the game has necessarily ended in a draw. Lyttleton (2016) analyses some 400 penalty shoot-outs in international and national competitions over a period of 20 years. He shows that the team that scored the last - in fact that got back into the game from a point of disadvantage during play - has a $61 \%$ probability of winning the shootout. The logic behind this is that since you got back into the game, you cannot lose, which reduces the team players' anxiety. Another factor that can reduce anxiety or improve confidence is for a player to score during the game. 
Jordet (2009) also investigates the probability of the best players scoring in penalty shootouts. Taking a sample of 41 "superstars", i.e. players rewarded for their individual performance in the FIFA competition, he shows that they are more likely to fail during penalty shoot-outs: they only score $65 \%$ of their penalties as opposed to nearly $74 \%$ for the other players. He hypothesizes that: (1) the most talented players are under more pressure because of their status, and (2) fear of missing the shot makes them change the way they usually play and therefore increases the failure rate. Given these facts, trainers should place the best players fifth up in a penalty shoot-out. This remark ties in with the findings of McGarry and Franks (2000): their computer simulation suggests that there is an advantage to placing the team's fifth-best penalty taker on the first kick, the fourth-best on the second kick, and so on until the last kick.

Whatever trainers or players choose, Jordet et al. (2007) find that the percentage of penalties scored is $86.6 \%$ by the first kicker, $81.7 \%$ by the second kicker, $79.3 \%$ by the third kicker, $72.5 \%$ by the fourth kicker, $80 \%$ by the fifth kicker and $64.3 \%$ thereafter. Unless the pressure is intense, this does not appear to fit the scenario suggested by the simulation. Not surprisingly, they also find that attackers successfully convert $83.1 \%$ of their penalties, midfield players convert $79.6 \%$ and defenders convert $73.6 \%$. Moreover, older players could be expected to have the advantage by force of habit. Yet players aged 22 or less successfully convert $85.2 \%$ of attempts, whereas those aged 23 to 28 convert $77.6 \%$, and those aged over 29 years convert $78.1 \%$.

Billsberry et al. (2007) analyze the effects of national cultural characteristics on the outcomes of penalty shoot-outs for 16 countries. They build different indicators of cultural dimensions. In particular, they study the cultural dimension of individualism/collectivism on penalty shoot-out outcomes. Their results show that the nations they call "collectivist" succeed better than the "individualists". The authors suggest that players from individualist nations are more anxious and under greater stress due to the blame they will attract if they miss. This could explain why English players fail in penalty shoot-outs, as Jordet (2009) writes. In his study, he selects the eight most merited European nations and obtains videos of penalty shoot-outs in World Cups and European Championships from 1976 to 2006. He analyses 200 shots taken by players representing these teams (Czech Republic, Denmark, England, France, Germany, Italy, Netherlands and Spain). He shows that players from countries at the top end of the Champions League - a proxy for team status - perform worse than other players, with the country representing this idea best being England. 
Greenlees et al. (2008) investigate the effect of a penalty kicker wearing a red shirt on the probability of scoring. Indeed, several studies have shown that wearing red improves sporting results (Hill and Barton, 2005; Attrill et al. 2008). The authors took a sample of 12 goalkeepers to whom they showed a video in which four players were about to take a penalty. The goalkeepers were given the same perspective as in a real match. Before shooting, every player was filmed with a different shirt color and the goalkeepers were asked to evaluate their chances of stopping the ball. The results show that players in red are seen by goalkeepers as being more capable of scoring.

\subsection{Should your team shoot first in a shoot-out?}

Apesteguia and Palacios-Huertas (2010) also show that the outcome of penalty shoot-outs depends on being the first team to shoot: the teams shooting first have a .60 probability of winning the shoot-out. Extending the study by increasing the sample of shoot-outs (from 129 to 540), Kocher et al. (2012) do not replicate Apesteguia and Palacios-Huertas' results: the teams shooting first have less chance of winning the shoot-out with a probability of .53 , not statistically different from .50. Palacios-Huertas (2014) in turn raises the sample to 1,001 penalty shoot-outs and confirms his first result.

Feri et al. (2013) carefully designed an experimental protocol with the help of the basket-ball teams to study the question of the first mover advantage or dis-advantage. They use series of free kicks with relatively (compared with the previous studies) low stakes. In this seeing they confirm that there is no first mover advantage.

Vandebroeck et al. (2016) develop a theoretical model to help understand why the empirical studies on soccer disagree. They first argue that psychological pressure raises the probability of the first team up winning the shoot-out: the first team has an advantage due to the presence of a lagging-behind effect. Second, they show that the samples used - notably by Kocher et al. (2012) - are too small to find a significant effect when the probability of winning is .53 . In effect, at the $5 \%$ (1\%) level, a unilateral test between .53 and 0.5 requires more than 750 $(1,500)$ observations to be significant.

So other studies are needed. In our paper, we are particularly interested in this hypothesis of "first mover advantage": playing on the first team to take the penalty shoot-out can (or cannot) provide a real advantage. 


\section{Rules, data and first results}

A penalty shoot-out is simply a sequence of penalty kicks. Penalty shoot-outs were introduced by football's international governing body, the Fédération Internationale de Football Association (FIFA), in 1970 to determine the winner in knockout tournament games in which there is a draw between two teams after 90 minutes of regulation playing time and 30 minutes of extra time. Prior to 1970 , the winner of a tied match in a knockout tournament was decided by a coin toss or a replay.

For example, a coin toss qualified Italy for the European Championship final following its semi-final draw against the USSR. History will recall that the first penalty shoot-out took place in England between Hull City and Manchester United during the semi-final of the Watney Cup (1-1 at the end of regulation playing time) and that the first kicker to score his penalty kick was none other than the illustrious George Best.

\subsection{The rules}

The rules that govern a penalty kick are described in the Official Laws of the Game. First, each team selects five players (from the players on the pitch in the $120^{\text {th }}$ minute). Second, the teams take the kicks alternately. Third, if before both teams have taken five kicks, one has scored more goals than the other could score, even if it were to complete its five kicks, no more kicks are taken. If, after both teams have taken five kicks, both have scored the same number of goals, the teams continue kicking in the same alternating order until one team has scored one goal more than the other from the same number of kicks (i.e. in a sequential oneon-one competition). Each penalty kick during the shoot-out must be taken by a different player, and all eligible players must have taken a kick before any player can take a second kick. In principle, this sequence can go on indefinitely. However, the longest shoot-out in our dataset is 22 kicks, i.e. 11 for each team. From 1970 to June 2003, the team that won the referee's toss of the coin before the shoot-out had to take the first kick. In July 2003, FIFA changed the rules by giving the winner of the referee's coin toss the option of choosing whether to kick first or second. However, winners of the coin toss almost always choose to go first.

\subsection{The sample: French cups}

The data come from French cup competitions the football association (FA) Cup ("Coupe de France"), the League Cup ("Coupe de la Ligue") and the Champions Trophy ("Trophée des champions"). The FA Cup is a national competition open to all amateur and professional fédération française de football (FFF)-member clubs. The League Cup is only open to 
professional clubs (in Ligue 1 and Ligue 2, as well as some third league National clubs). The dataset comprises 252 penalty shoot-outs: 135 for the FA Cup in the 2007-2017 period, 110 for the League Cup in the 2001-2018 period and all the Champions Trophy shoot-outs since it was first played (1995). Only 239 of these 252 shoot-outs contain the full details on them, totalling 2,504 penalty kicks. These data were collected from different sites, including the LFP (French Professional Football League) and the L'Equipe newspaper.

For every shoot-out in every competition, we have information on the date, the identity of the teams kicking first and second, the final outcome of 252 penalty shoot-outs, the outcomes of each of the kicks in the sequence for 239 penalty shoot-outs (with the players' names), the number of people in the stadium, and the geographical location of the match (that is, whether the game was played at home, away or on a neutral ground). Table 2 displays these statistics.

Table 2: Description of the dataset

\begin{tabular}{lccc}
\hline \hline & Seasons & $\begin{array}{c}\text { Penalty } \\
\text { shoot-outs }\end{array}$ & $\begin{array}{c}\text { Penalty } \\
\text { kicks (239 } \\
\text { shoot-outs) }\end{array}$ \\
\hline Competition & $2007-2017$ & 135 & $N$ \\
FA Cup & $2007-2017$ & 46 & \\
$\quad$ Round of 128 & $2007-2017$ & 52 & \\
Round of 64 & $2007-2017$ & 21 & \\
Round of 32 & $2007-2017$ & 8 & \\
Round of 16 & $2007-2017$ & 8 & \\
Quarter & $2001-2018$ & 110 & 1141 \\
League Cup & $2001-2018$ & 53 & \\
$\quad$ Preliminary rounds & $2001-2017$ & 26 & \\
Round of 32 & $2001-2017$ & 16 & \\
Round of 16 & $2001-2017$ & 10 & \\
Quarter & $2001-2017$ & 4 & \\
Semi & $2001-2017$ & 1 & \\
Final & $1995-2017$ & 7 & \\
Champions Trophy & & & \\
& & 252 & \\
\hline Total & & & \\
\hline \hline
\end{tabular}

Source: LFP

\subsection{Empirical evidence}

As mentioned above, an empirical controversy is currently raging over the existence of any possible psychological pressure bearing on kicking second in penalty shoot-outs. Palacios- 
Huerta and his co-authors $(2010,2014,2016)$ find that there is a psychological advantage to kicking first, since the term starting the shoot-out has a $60 \%$ chance of winning it as opposed to $40 \%$ for the team kicking second. Yet this finding is challenged by Kocher et al. (2012), who find no correlation between starting a penalty shoot-out and winning it (53\% vs. 47\%, with the difference being non-significant). Since neither of the two studies collects together data on France, we have undertaken this empirical work.

Figure 1: Winner Frequencies in Aggregate Data

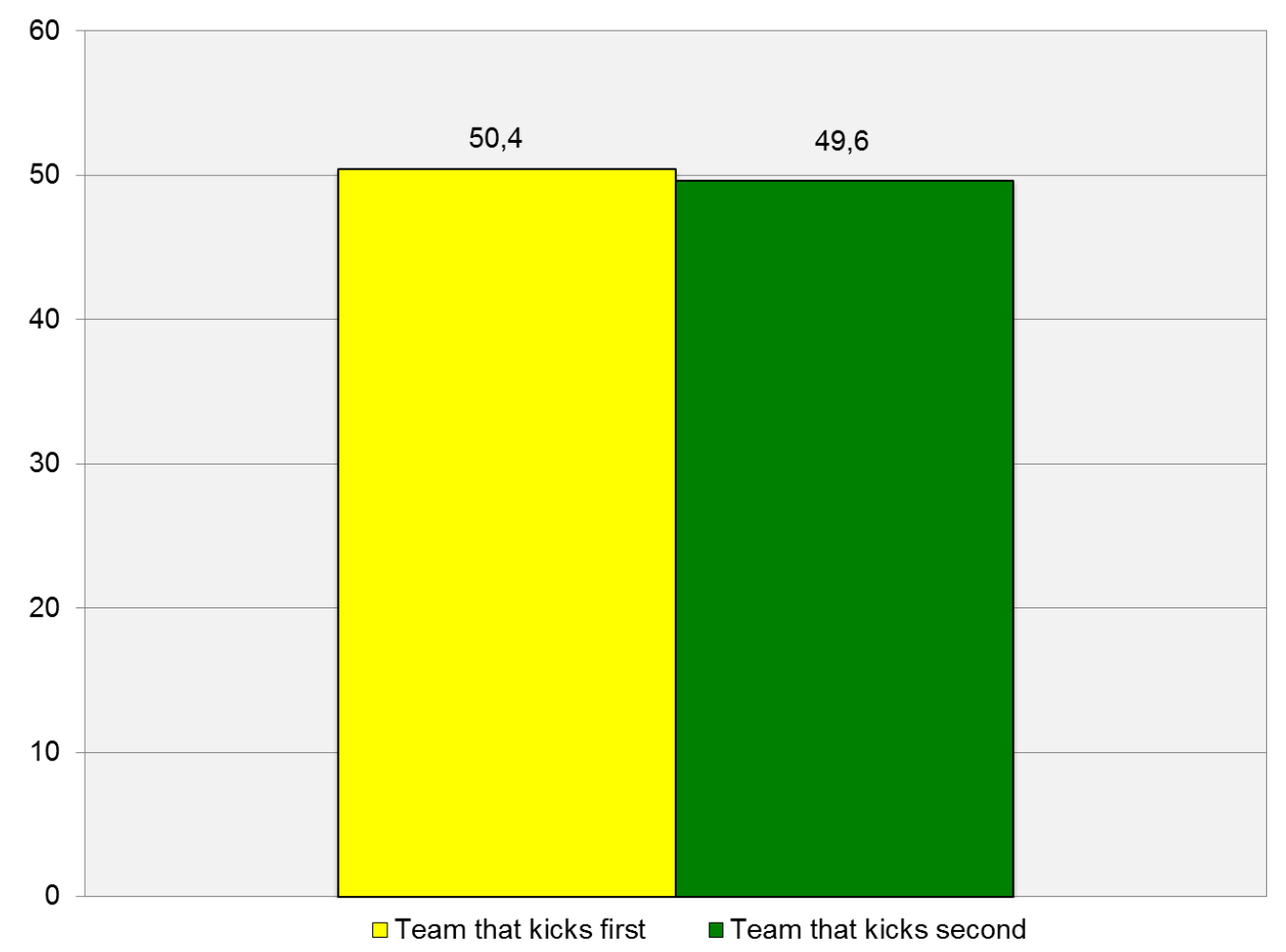

Source: LFP

Figures 1 and 2 present the descriptive statistics calculated for the abovementioned sample. The results are by and large even closer than the findings by Kocher et al. (2012): we obtain a more or less equiprobable breakdown of winners between the teams that start the penalty shoot-out and those that kick second: $50.4 \%$ vs. $49.6 \%$.

The results do not change significantly with the competition: the breakdown for the FA Cup is $51.1 \%$ / $48.9 \%$ and the breakdown for the League Cup is $49.1 \%$ / 50.9\%. None of these ratios is statistically different from the 50\% / 50\% breakdown (Table 3). To sum up, our results support the findings of the study by Kocher et al. (2012) rather than the results of Palacios and his co-authors (2010, 2014 and 2016). This therefore suggests that there is no advantage, in France, to starting a penalty shoot-out... 
Figure 2: Winner Frequencies by Cup

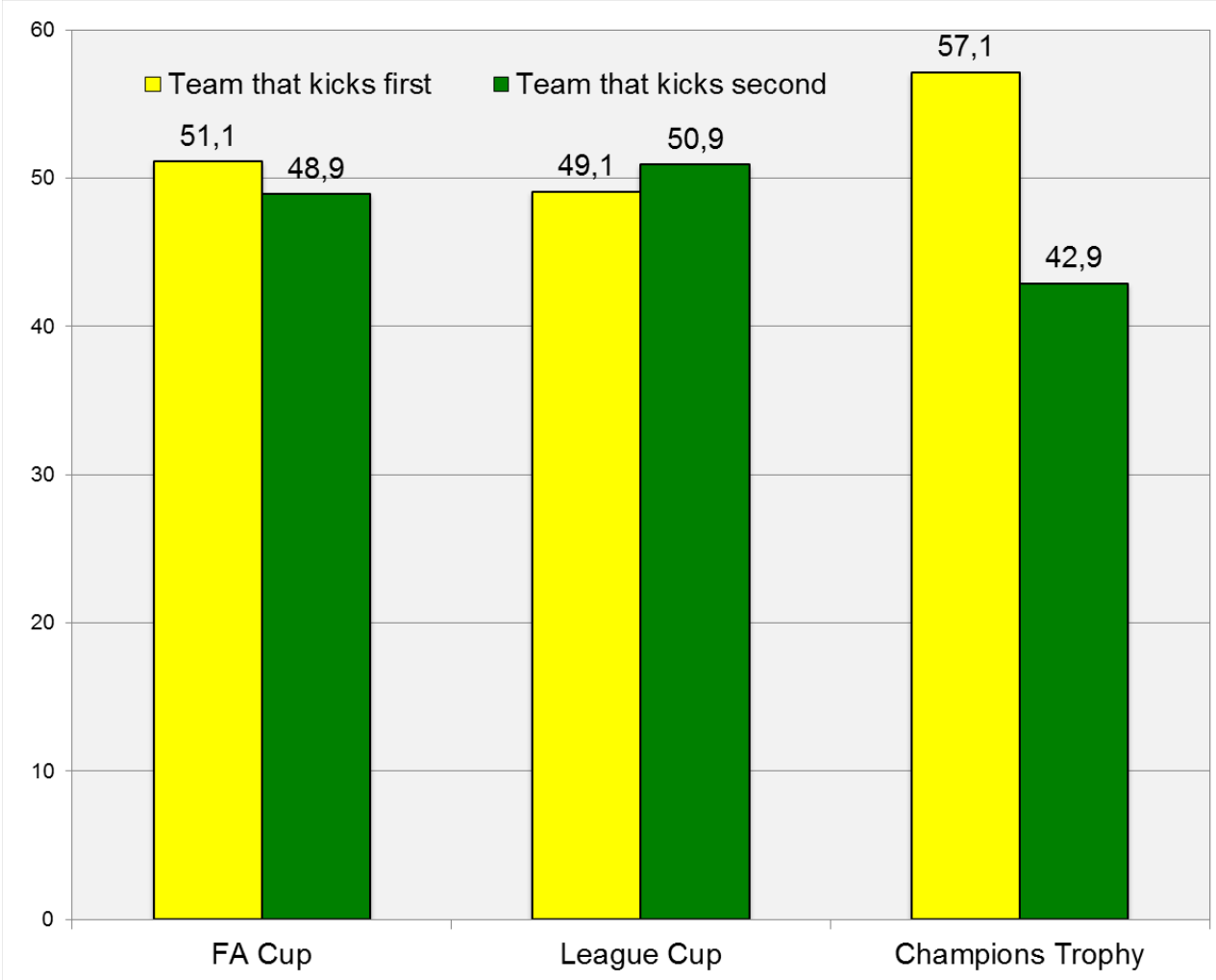

Source: LFP

Table 3: Percentage of winners among the teams kicking first

\begin{tabular}{lccc}
\hline \hline & $\begin{array}{c}\text { Number of } \\
\text { shoot-outs }\end{array}$ & $\begin{array}{c}\text { \% first team } \\
\text { wins }\end{array}$ & $\begin{array}{c}\text { p-value (bilateral } \\
\text { binomial test) }\end{array}$ \\
\hline FA Cup & 135 & 51.1 & 0.86 \\
League Cup & 110 & 49.1 & 0.92 \\
Champions Trophy & 7 & 57.1 & 1.00 \\
Total & $\mathbf{2 5 2}$ & $\mathbf{5 0 . 4}$ & $\mathbf{0 . 9 5}$ \\
\hline
\end{tabular}

Source: LFP

Table 4 presents the estimates of the probability of winning the penalty shoot-out (linear probability model and Probit model) for the sample of teams based on a number of explanatory factors, notably the number of kicks. The main result is borne out: kicking first has no impact on the outcome of the penalty shoot-out, even when other variables are taken into account (home match and level of the team). Of these additional variables, only the level of the team influences the probability of winning the shoot-out, while the fact of playing home or away has no effect. More specifically, we note that a team at a higher level than its opponent has an approximately $20 \%$ larger probability of winning the penalty shoot-out. 
Table 4: Determinants of Winning the Tournament

\begin{tabular}{lcccc}
\hline \hline & Lpm & Lpm & Probit & Probit \\
\hline Constant & $0.496^{* * *}$ & $0.452^{* * *}$ & -0.001 & -0.116 \\
& $(0.032)$ & $(0.042)$ & $(0.079)$ & $(0.106)$ \\
The team kicks first & 0.008 & 0.006 & 0.020 & 0.015 \\
& $(0.045)$ & $(0.045)$ & $(0.112)$ & $(0.113)$ \\
Home match & & 0.028 & & 0.067 \\
& & $(0.048)$ & & $(0.120)$ \\
Neutral ground & & 0.012 & & 0.087 \\
& & $(0.193)$ & & $(0.348)$ \\
Higher level & & $0.133^{* *}$ & & $0.320 * *$ \\
& & $(0.057)$ & & $(0.139)$ \\
Fixed effects for: & & & & \\
FA Cup & & & No & No \\
League Cup & Yes & Yes & No & No \\
Champions Trophy & Yes & Yes & Yes & No \\
\hline N (teams) & & & 504 & 504 \\
R2(Pseudo) & 504 & 504 & 0.000 & 0.010 \\
\hline \hline
\end{tabular}

\section{Does the kicker have other anxieties? The analysis of penalty shoot-outs}

Given the above results, it would appear that, contrary to the findings of Palacios and his colleagues, the fact of starting a penalty shoot-out procures no psychological advantage liable to influence the final outcome. The 60/40 hypothesis therefore arguably has no empirical substance. Therefore, the question must be raised again: Does the outcome of a shoot-out depend solely on the skill of the kickers or are "emotions" required to explain the success of a penalty shoot-out?

The purpose of this section is to test different hypotheses regarding the player's "anxieties" at the moment of the penalty kick. We start (4.1.) with variables already considered in the literature regarding football penalty shoot-outs. We then turn (4.2.) to variables derived from general decision theory under uncertainty. 
Figure 3: Scoring Probability Per Round

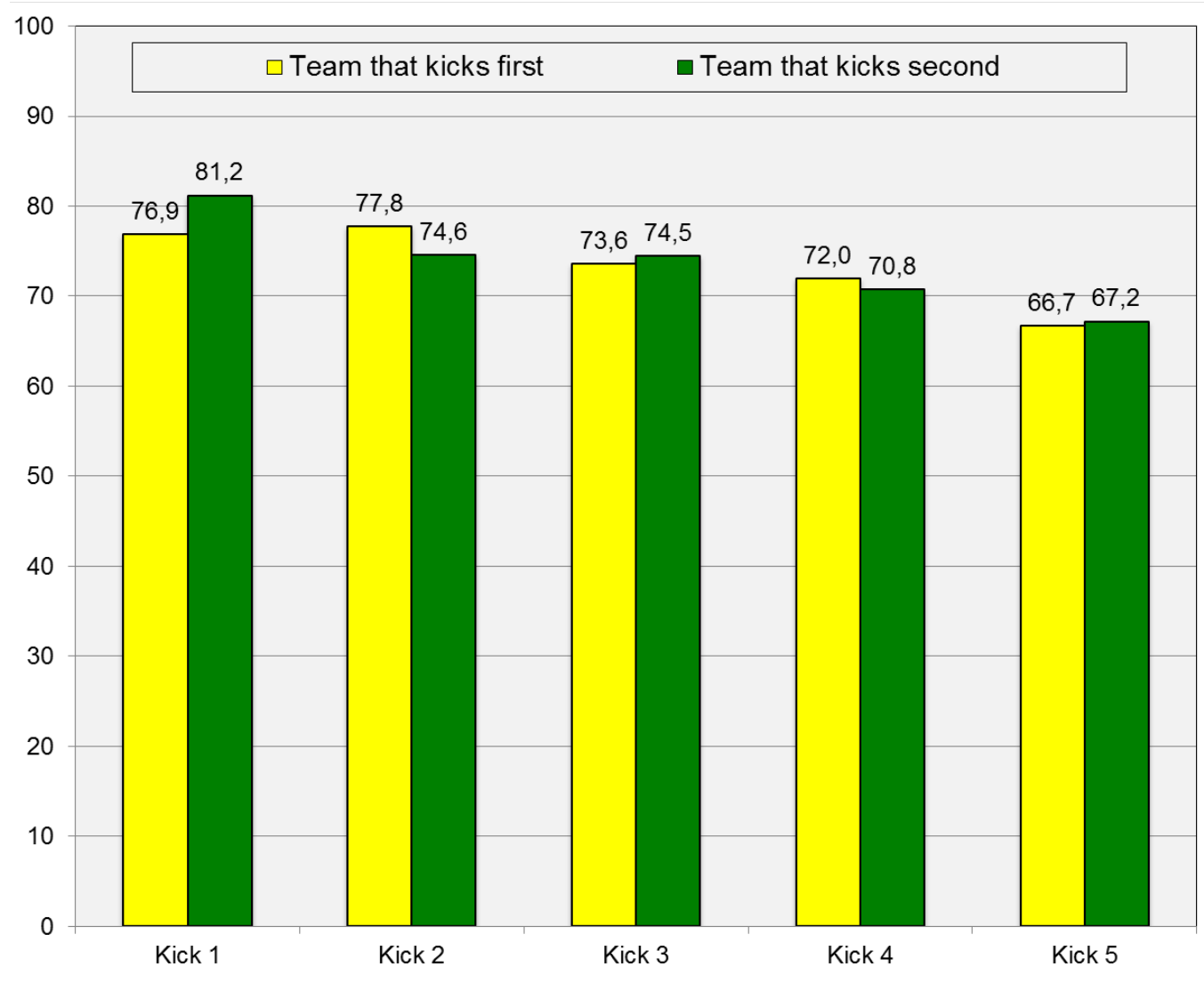

Source: LFP

\section{1. "Survival”, “Catch up”, "Break point”...}

We have managed to fully reconstitute 239 penalty shoot-outs of the 252 shoot-outs in the sample. Figure 3 presents the percentages of success for the different kicks in the shoot-out. Our data largely support two usual findings:

1) The success rate is lower during shoot-outs than during the game: $73.1 \%$ vs. more than $76 \%$.

2) The probability of scoring the kick decreases along the shoot-out sequence: nearly $80 \%$ for the first round as opposed to less than $70 \%$ for the fifth round.

These two findings can be explained quite simply by the fact that not all the players are shootout specialists and that the teams generally put up their best kickers (1) during the game and (2) at the beginning of the shoot-out. However, our shoot-outs reveal no significant differences in any round between the shoot-out successes of players on the starting team and players on the second team.

This result is also found for the analysis of the teams in the lead in each different round (Figure 4). Unlike the study by Palacios and his colleagues, we do not find that being first up 
provides any advantage in terms of leading the shoot-out. We even find the opposite to be the case for the first round! At the end of the first round, the team first up is in the lead in $12.1 \%$ of cases, whereas the teams kicking second are in the lead in $16.3 \%$ of cases (both teams are level pegging in $72.6 \%$ of the shoot-outs). Note that approximately $30 \%$ of penalty shoot-outs continue past the five rounds and therefore proceed to "sudden death".

Figure 4: Frequency with which a team leads in the score at the end of a round

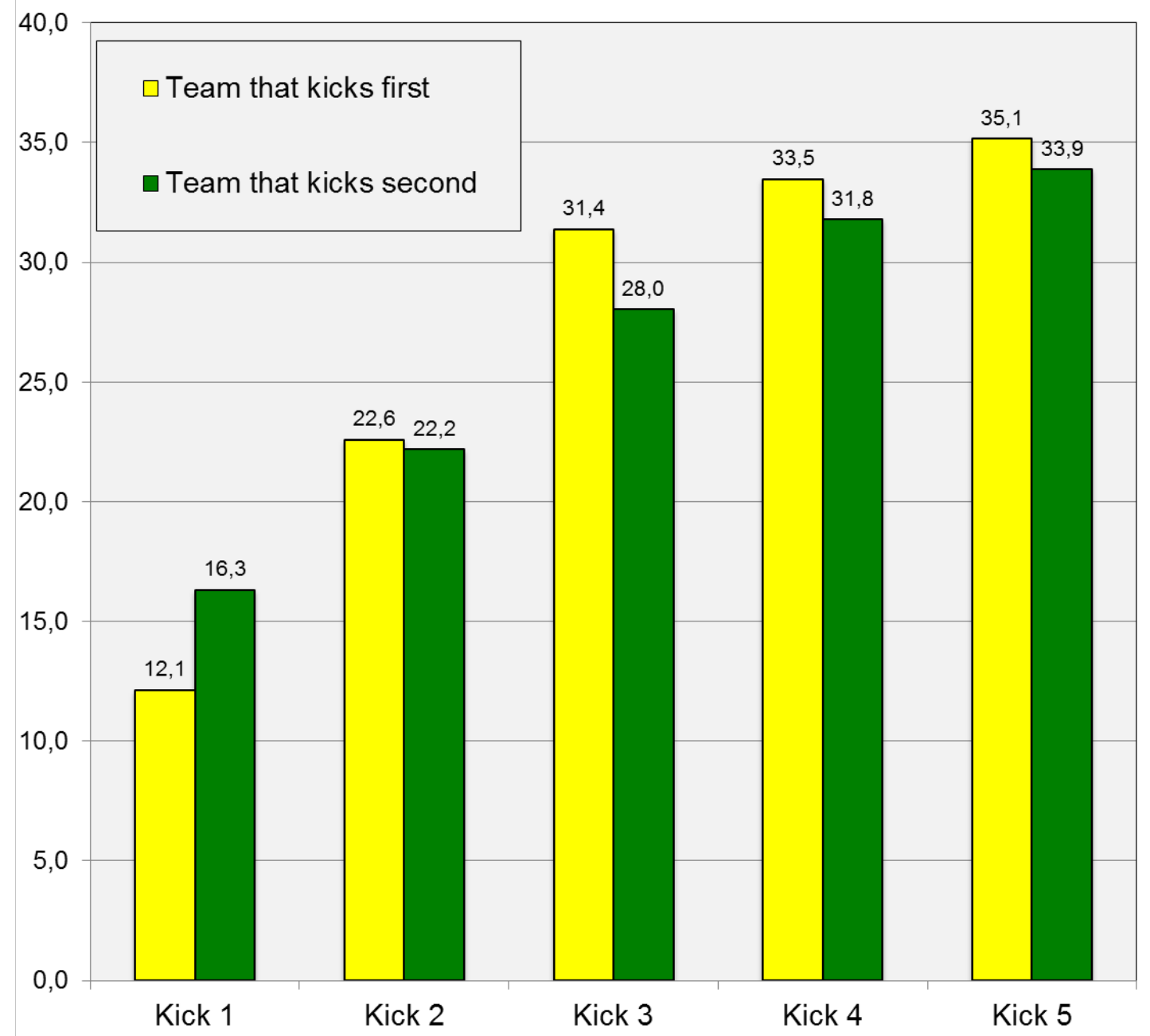

Source: LFP

Again, our data do not appear to support the hypothesis put forward by Palacios and his coauthors whereby the first kicker has a higher probability of success. Our first hypothesis is simply a variation on the theme. It amounts to assuming that the psychological pressure of the "lag" does not come into play in the first round, but only when the number of kicks is identical. So the second team's first kicker would not feel any particular emotion about coming after the other team's first kicker, even if the latter had scored. If, on the other hand, a 
team lags behind when the number of kicks is the same for both teams, the kicker would then feel pressured due to the fact that missing his kick would raise the risk of causing his team to lose. To test this hypothesis, we need to analyze the determinants of a successful kick.

The test of the hypothesis by Palacios and his co-authors consists simply of testing the negative effect of lagging behind in goals on the player scoring his own kick ( $Y=0$ or 1$)$, considering or not other characteristics $(X)$. If the probability of scoring is denoted $p$, the following econometric model is estimated:

$$
p(Y=1)=F\left(p_{0}+\alpha \Delta B u t+\beta X\right)
$$

where $p_{0}$ is a constant, $\alpha$ and $\beta$ are the coefficients to be estimated and $F($.$) is the distribution$ function.

Our variation is then rather to test the effect of a lag for the same number of kicks on scoring or missing, i.e. the model:

$$
p(Y=1)=F\left(p^{\prime}{ }_{0}+\alpha^{\prime}[\Delta B u t(+1 \text { or } 0)]+\beta X\right)
$$

We add +1 goal to the number of goals for the team that is second up to kick (none for the team starting the shoot-out).

Table 5 presents the results of the estimations of the probability of scoring a kick based on the score difference, with or without lag correction. Two techniques were used for function $\mathrm{F}($.$) :$ the linear model and the Logit one, both with fixed effects. The estimations confirm that the mere score difference has no significant effect, unlike the difference adjusted for the number of kicks which has a negative impact on the probability of scoring. Note that this probability also depends negatively on the kick order number in the shoot-out, as we observed in the descriptive statistics: different pressure or less-skilled players...

A more sophisticated specification then consists in combining the above two hypotheses by considering three situations. The first situation is a "catch-up kick", as it were, which corresponds to Palacios's hypothesis, when the player kicks with just one goal down, but also when one penalty kick less has been taken. The second concerns a situation where, for the same number of penalty kicks, the kicker's team is one goal ahead ("break-point kick"). Conversely, the third situation considers the kicker of a team that, for the same number of penalty kicks, is one goal down (“survival kick”). 
Table 5: Probability of scoring a penalty kick

\begin{tabular}{lcccc}
\hline \hline & Lpm & Logit & Lpm & Logit \\
& FE & FE & FE & FE \\
\hline & & & & \\
Difference in goals & -0.001 & -0.041 & & \\
& $(0.013)$ & $(0.067)$ & & \\
& & & & $0.370^{* * *}$ \\
Difference in goals & & & $0.083 * * *$ & $(0.065)$ \\
(equal number of kicks) & & & $(0.013)$ & $-0.170 * * *$ \\
& & & & \\
Rank & $-0.031 * * *$ & $-0.163 * * *$ & $-0.032 * * *$ & \\
& $(0.005)$ & $(0.028)$ & $(0.005)$ & 2504 \\
& & & & 2504 \\
\hline N (kicks) & 2504 & 2504 & 239 & 239 \\
N (shoot-outs) & 239 & 239 & & \\
\hline \hline
\end{tabular}

Figure 5 presents the raw probabilities of scoring the kick based on these scenarios: the kicker's team leads for an equal number of penalty kicks ("break-point kick") or conversely is at a disadvantage ("survival"), or even has the possibility of equalizing ("catch up"). We observe that if there exists a psychological advantage, it is not about being "under pressure" (70.2\% kick success rate) or playing for "survival" (70.8\% rate), but rather about the "comfort" of having a goal advantage $(83.6 \%$ rate). Here again, the hypothesis of the advantage of being the first up to kick does not emerge from the statistics.

These raw effects are corroborated by the econometric estimations in Table 6, which even reveal that the probability of scoring is lower when playing a simple catch-up kick than when playing for survival. A parallel could be drawn here with studies regarding the influence of "stress" on risk-taking, which find that individuals prove more likely to take risks in the loss "domain", but more "conservative" in the gain "domain" (Porcelli and Delgado, 2009). Kicker anxiety is therefore much more complex than the mere "catch-up" hypothesis would have it. In the following section, we propose other emotional mechanisms inspired by action theory that could well explain a successful penalty kick. 
Figure 5: Scoring probability according to scenario (survival/catch up/break point)

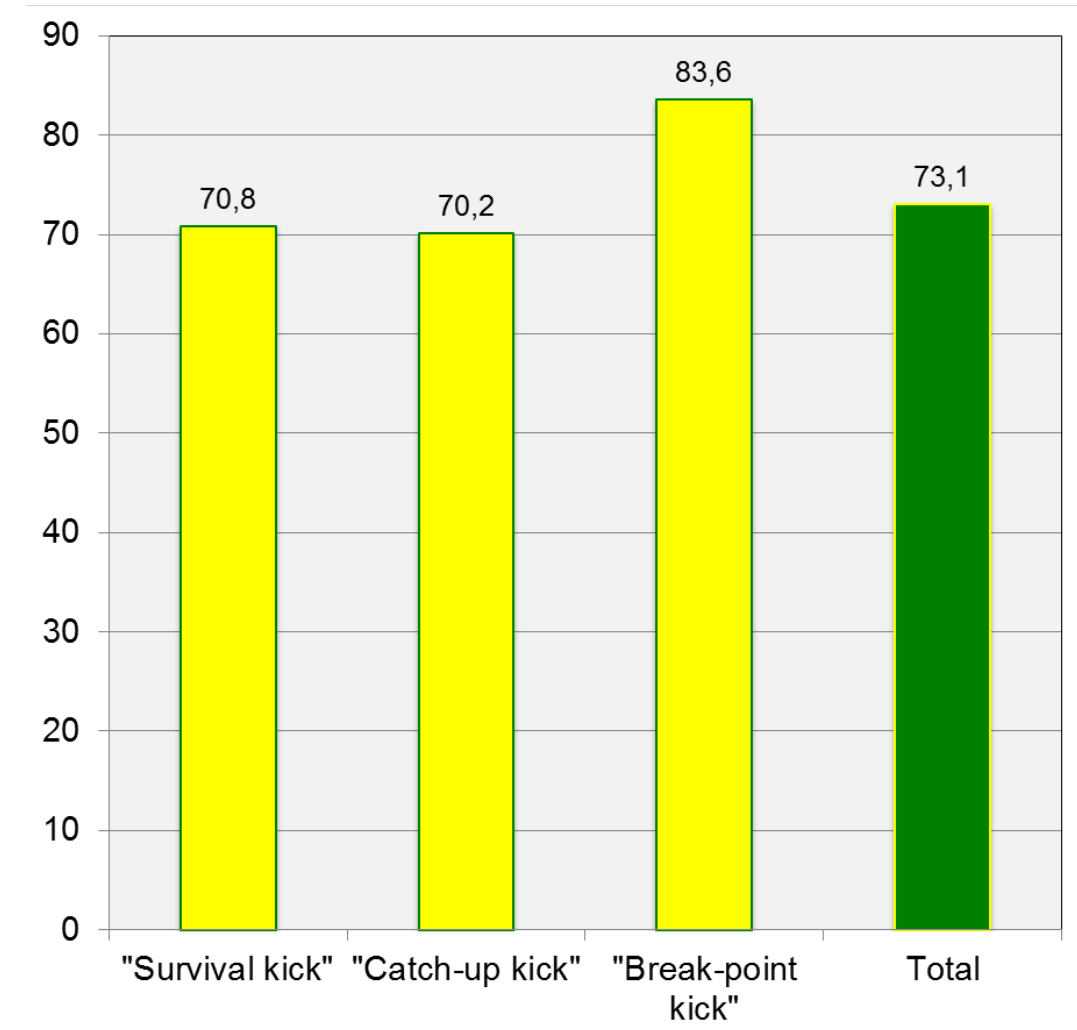

Source: LFP

Table 6: Probability of scoring a kick in a shoot-out

\begin{tabular}{lcc}
\hline \hline & Lpm & Logit \\
& FE & FE \\
\hline \multirow{3}{*}{ Survival } & & \\
& $-0.112^{* * *}$ & $-0.663 * * *$ \\
& $(0.030)$ & $(0.17)$ \\
Catch-up & & \\
& $-0.249^{* * *}$ & $-1.340 * * *$ \\
& $(0.024)$ & $(0.146)$ \\
Rank & & \\
& $-0.041^{* * *}$ & $-0.220 * * *$ \\
& $(0.005)$ & $(0.030)$ \\
\hline N (kicks) & & \\
N (shoot-outs) & 2504 & 2504 \\
\hline \hline
\end{tabular}

Source: LFP

\section{2. "Pressure" and "stake"...}

This section constructs two variables directly inspired by the theory (Kahneman and Tversky 1979). In most cases, even if a player scores his penalty kick, there is no certainty that his 
team will ultimately win. Likewise, if he fails, it is not certain that the team will lose. So the ultimate consequences of the action of kicking are uncertain. As with all uncertainty, whether probabilistic or not, the kicker addresses this uncertainty on the basis of two main aspects: the importance of the stake and the associated risk. In the following, we consider a simple probabilistic model to quantify these two aspects.

A penalty shoot-out can be regarded as a binary tree, where each node is the system state at the moment a team kicks with the possible kicks it can take and is therefore followed by two nodes corresponding to the two possible outcomes (success/failure) of this kick, except for the terminal nodes where one team has won.

Attached to each terminal node is a utility vector describing the victory, which is therefore simply +1 for the winning team and 0 for the losing team. The probability of the kick in question scoring can be attached to each non-terminal node. For example, the simplest model considers that each kick scores with the same probability. By means of simple recursive multiplication working back from the terminal nodes, we can deduce three other probabilities for each node: the probabilities that the kicker's team ultimately wins (1) before the kick, (2) if the kick scores, and (3) if the kick fails.

We have therefore considered all the possible penalty shoot-out sequences and generated different probabilities with respect to the probability, assumed constant, of scoring one's penalty kick $(p=0.731)$ and retaining an equiprobable probability of winning $(0.5)$ in the case of sudden death (see Appendix 1). We have calculated the three probabilities at each step of the penalty shoot-out, and according to the sequence: the probability of winning the penalty shoot-out before kicking $\left(\mathrm{p}_{0}\right)$, the probability of winning if the kick scores $\left(\mathrm{p}_{+}\right)$and the probability of winning if the kick does not score (p_).

Table 7 illustrates the calculation of these probabilities based on two penalty shoot-outs observed in our sample. In the first shoot-out, Team A starts and wins the shoot-out after nine penalty kicks. After the eighth penalty kick, Team A is leading with score 3-2. If the team scores, it wins the match $\left(\mathrm{p}_{+}=1\right)$; if it does not score, Team B will have another penalty kick to take and the probability of Team A winning the shoot-out decreases to $p_{-}=0.6345$; and lastly, the probability of the team winning the match at the moment of the ninth penalty kick, given that the score is $3-2$ to its advantage, is equal to $\mathrm{p}_{0}=0.901681$, which is computed as $0.731 * 1+0.269 * 0.6345$. 
For the second penalty shoot-out reported in Table 7, both teams are tied after the tenth penalty kick and have to go to sudden death to decide the match. Before taking its fifth penalty kick, Team B is behind with score 3-4. If Team B scores, the probability of Team B winning the match is 0.5 ( $\mathrm{p}_{+}$as initially), but if it fails, it loses the match $\left(\mathrm{p}_{-}=0\right)$; the probability of Team B winning the match conditionally on the score is $\mathrm{p}_{0}=0.3655$ (before kicking).

Table 7: Stake and pressure

\begin{tabular}{|c|c|c|c|c|c|c|}
\hline Sequence & Score befo & the kick & $\mathbf{p}_{\mathbf{0}}$ & $\mathbf{p}_{+}$ & $\mathbf{p}$ & $\begin{array}{l}\text { Scores } \\
\text { or not }\end{array}$ \\
\hline & Team A & Team B & & & & \\
\hline 1 & 0 & 0 & 0.500000 & 0.573479 & 0.300324 & 0 \\
\hline 2 & 0 & 0 & 0.699676 & 0.773155 & 0.500000 & 0 \\
\hline 3 & 0 & 0 & 0.500000 & 0.581247 & 0.300324 & 1 \\
\hline 4 & 1 & 0 & 0.418753 & 0.500000 & 0.197966 & 0 \\
\hline 5 & 1 & 0 & 0.802034 & 0.879322 & 0.592006 & 1 \\
\hline 6 & 2 & 0 & 0.120678 & 0.157972 & 0.019333 & 1 \\
\hline 7 & 2 & 1 & 0.842028 & 0.928128 & 0.608052 & 1 \\
\hline 8 & 3 & 1 & 0.071872 & 0.098320 & 0.000000 & 1 \\
\hline 9 & 3 & 2 & 0.901681 & 1.000000 & 0.634500 & 1 \\
\hline 1 & 0 & 0 & 0.500000 & 0.573479 & 0.300324 & 1 \\
\hline 2 & 1 & 0 & 0.426521 & 0.500000 & 0.226845 & 0 \\
\hline 3 & 1 & 0 & 0.773155 & 0.843775 & 0.581247 & 1 \\
\hline 4 & 2 & 0 & 0.156225 & 0.197966 & 0.042794 & 1 \\
\hline 5 & 2 & 1 & 0.802034 & 0.879322 & 0.592006 & 1 \\
\hline 6 & 3 & 1 & 0.120678 & 0.157972 & 0.019333 & 1 \\
\hline 7 & 3 & 2 & 0.842028 & 0.928128 & 0.608052 & 0 \\
\hline 8 & 3 & 2 & 0.391948 & 0.500000 & 0.098320 & 1 \\
\hline 9 & 3 & 3 & 0.500000 & 0.634500 & 0.134500 & 1 \\
\hline 10 & 4 & 3 & 0.365500 & 0.500000 & 0.000000 & 1 \\
\hline
\end{tabular}

Source: own calculation

By computing these probabilities, we are able to define the two emotions evidenced by action theory that are liable to influence the success of a penalty kick. The first one, we'll call "stake", is the difference between $p_{+}$and $p_{-}$: this stake is at its maximum when, for example, the penalty kick decides the outcome of the match (either $\mathrm{p}_{-}=0$ and $\mathrm{p}_{+}=0.5$, or $\mathrm{p}_{+}=1$ and 
$\left.\mathrm{p}_{-}=0.5\right)$. The second emotional parameter takes the form of "pressure" on the shooter and is quantified by the probability, before the kick, of losing the match $\left(1-\mathrm{p}_{0}\right)$. The ex-ante probability of losing obviously depends on the difference in the score. Take the case of the tenth kicker in the second shoot-out in Table 7: the "stake" of this penalty kick is at its maximum $\left(\mathrm{p}_{+}-\mathrm{p}_{-}=0.5\right)$ and the "pressure" is high $(0.735)$ because if the kicker fails, the match is lost. If the kicker is put off by these emotions, they will in principle lower the probability of scoring his penalty kick. The "stake" would be the same for a penalty kick with a prospective match win $\left(\mathrm{p}_{+}-\mathrm{p}_{-}=1-0.5=0.5\right)$, but there would be much less "pressure" because, in the event of failure, the game would not end (it would proceed to the "sudden death" phase). Empirically, the correlation between these two emotions in our sample is indeed negative, at approximately -0.33 .

Table 8: Probability of scoring a penalty kick

\begin{tabular}{lcc}
\hline \hline & Linear & Logit \\
& FE & FE \\
\hline Pressure & & \\
& $-0.154^{* * *}$ & $-0.714^{* * *}$ \\
& $(0.046)$ & $(0.231)$ \\
Stake & & \\
& $-0.601 * * *$ & $-2.929 * * *$ \\
& $(0.123)$ & $(0.621)$ \\
Rank & & \\
& $-0.024 * * *$ & $-0.149 * * *$ \\
& $(0.007)$ & $(0.040)$ \\
& & \\
\hline N (kicks) & 2169 & 2169 \\
N (shoot-outs) & 235 & 235 \\
Source: LFP & &
\end{tabular}

We have tested this behavioral model on the above-described data using panel regressions with fixed effects (Table 8). Given that the importance of the stake grows as a rule on progressing through the tree and that the teams put up their best players to kick first, the notso-good players consequently end up kicking when the stake is high. We have taken account of this effect by controlling by penalty kick order number in the shoot-out. The results show that "stake" and "pressure" are indeed two "emotions" that need to be taken into account to estimate the probability of scoring a penalty kick: "pressure", but also "stake", are emotional factors that reduce the probability of scoring. 
To illustrate our point, take the case of the linear probability model. The probability of scoring one's penalty kick estimated at average values is 0.739 . When the "pressure" is at its highest, this probability falls to 0.663 (other things being equal), and when the "stake" is highest, the probability is just 0.620 . If the penalty shoot-out were to go through to the end, the tenth player would have a 0.680 probability of scoring his penalty kick. This probability would be just 0.604 in the event of maximum "pressure" and 0.560 in the case of the stake being at its maximum.

\section{Conclusion}

Observation of penalty shoot-outs in football forms a remarkable natural individual decisionmaking experiment. Unlike certain microeconomic decision-making models, the kicker's action is not the result of a cost-benefit calculation between the effort made and the chances of a gain: this is the end of the match and kickers can "give their all". Yet the success of the action is tied in with what are defined as psychological factors. We have, in particular, shown that the importance, for the professional football player as for any one, of the two familiar dimensions of decision, the stake and the risk of losing, have a negative influence: even experienced shooters score less when the stake is high and when there is a greater risk of losing.

So what of the "goalie's anxiety at the penalty kick"? The work presented here has in effect totally disregarded the existence of the goalkeeper. Pressure is lower for goalkeepers in this exercise in which it is extremely rare to see them criticized for not saving a penalty kick. As Fabien Barthez, former goalkeeper for France, put it: "In this kind of exercise, the pressure is not on the goalkeeper, but on those who are going to take the kick." 


\section{References}

Ariely, D. (2008). Predictably Irrational, Revised and Expanded Edition: The Hidden Forces That Shape Our Decisions. Harper Collin, New York.

Apesteguia, J., and Palacios-Huerta, I. (2010), "Psychological pressure in competitive environments: evidence from a randomized natural experiment" American Economic Review. 100(5): 2548-2564.

Attrill Martin, Karen Gresty, Russel Hill and Robert Barton (2008), Red shirt colour is associated with long-term team success in English football. Journal of Sports Sciences, 26, 577-582.

Azar, O. H., and Bar-Eli, M. (2011), "Do soccer players play the mixed-strategy Nash equilibrium?", Applied Economics, 43(25), 3591-3601

Bar-Eli, M., Azar, O. H., Ritov, I., Keidar-Levin, Y. and Schein, G. (2007), “Action bias among elite soccer goalkeepers: The case of penalty kicks", Journal of Economic Psychology, 28(5), 606-621.

Bellock, S. L. and R. Gray (2007), “Why do atheletes choke under pressure?” in: G. Tenenbaum and R. C. Eklund (eds.) Handbook of Sports Psychology. Wiley and sons, 425-444.

Billsberry, J., Nelson, P., Van Meurs, N. and Edwards, G. (2007), "Are penalty shoot-outs racist?", Journal of Sports Science and Medicine, 6(10), 98.

Botwell, M., King, M.A. and Pain, M.T.G., (2009), "Analysis of the keeperdependent strategy in the soccer penalty kick", International Journal of Sports Science and Engineering, 3 (2), 93-102.

Chiappori, P. A., Levitt, S. and Groseclose, T. (2002), "Testing mixed-strategy equilibria when players are heterogeneous: The case of penalty kicks in soccer", American Economic Review, 1138-1151.

Dohmen, T. J. (2008), "Do professionals choke under pressure?", Journal of Economic Behavior and Organization, 65, 636-653.

Feri, F., Innocenti A. and P. Pin (2013), "Is there psychological pressure in competitive environments?", Journal of Economic Psychology, 39: 249-256.

Genakos, C. and M. Pagliero (2012), "Interim rank, risk taking, and performance in dynamic tournaments", Journal of Political Economy, 120(4), 782-813.

Gonzalez-Diaz, J and I. Palacios-Huerta (2016), "Cognitive performance in competitive environments: Evidence from a natural experiment", Journal of Publics Economics 139: 40-53

Greenlees, I., Leyland, A., Thelwell, R. and W. Filby, (2008), “Soccer penalty takers' uniform colour and pre-penalty kick gaze affect the impressions formed of them by opposing goalkeepers", Journal of Sports Sciences, 26(6), 569-576.

Guiso L. and P. Sodini (2013). Household Finance: An Emerging Field, in G. M. Constantinides, M. Harris and R. M. Stulz, Handbook of the Economics of Finance, chapter 21.

Hickman, D. C. and N. E. Metz, (2015), "The impact of pressure on performance: Evidence from the PGA TOUR" Journal of Economic Behavior and Organization $116,319-330$. 
Hill, D. M., S. Hanton, N. Matthews and S. Fleming (2013), "Choking in sports: a review” International Review of Sport and Exercise Psychology 3(1), 24-39.

Hill R. and R. Barton (2005), "Red enhances human performance in contests", Nature, 435, p. 293.

Jordet, G., Hartman, E., Visscher, C. and K. A. Lemmink (2007), "Kicks from the penalty mark in soccer: The roles of stress, skill, and fatigue for kick outcomes", Journal of Sports Sciences, 25(2), 121-129.

Jordet, G., and M. T. Elferink-Gemser, (2012), "Stress, coping, and emotions on the world stage: The experience of participating in a major soccer tournament penalty shootout", Journal of Applied Sport Psychology, 24(1), 73-91.

Jordet, G., Elferink-Gemser, M. T., Lemmink, K. A. and C. Visscher (2006), "The" Russian roulette" of soccer? Perceived control and anxiety in a major tournament penalty shootout", International Journal of Sport Psychology, 37(2/3), 281-298.

Jordet, G. (2009), "Why do English players fail in soccer penalty shootouts? A study of team status, self-regulation, and choking under pressure", Journal of Sports Sciences, 27(2), 97-106.

Kahneman, D., and A. Tversky (1979), "Prospect theory: An analysis of decision under risk", Econometrica, 47, 263-291.

Kocher, M., Lenz, M.V. and M. Sutter (2012), "Psychological pressure in competitive environments: new evidence from randomized natural experiments", Management Science, 58, 1585-1591.

Lyttleton, B. (2016). Twelve Yards. Random House.

Kuss, O., Kluttig, A. and O. Stoll (2007), "The fouled player should not take the penalty himself": An empirical investigation of an old German football myth", Journal of Sports Sciences, 25(9), 963-967.

McGarry T. and I. M. Franks (2000), "On winning the penalty shoot-out in soccer", Journal of Sports Sciences, 18:6, 401-409,

Palacios-Huerta, I. (2014), Beautiful Game Theory: How Soccer Can Help Economics. Princeton University Press.

Vandebroek, T. P., McCann, B. T., and G. Vroom (2016), "Modeling the Effects of Psychological Pressure on First-Mover Advantage in Competitive Interactions. The Case of Penalty Shoot-Outs", Journal of Sports Economics, to be published. 


\section{Appendix}

\subsection{Benchmark case for the probability of winning}

The probability of team A to eventually win depends, in a rather complicated way, of where one stands in the sequence of penalty kicks: how kicks have already occurred, what is the score, and what are the probabilities of scoring of the shooters to come. In this section we compute these probabilities under the simple hypothesis that any player, at any time, scores with a fixed probability $p$.

Let $t \in\{1, \ldots, 10\}$ be the index for the first 10 shoots. Let $h$ be a possible history of 10 shots; for instance $h=(0,1,0,0,0,0,0,0,0,0)$ is the sequence such that only the first shoot of the second team is scored. Observe that this particular history will in fact never be seen because after the tenth shoot of the first team, the score is $(0,1)$ so the second team already wins. But it will simplify notation to consider that all histories runs up to 10 kicks, even if some of them are virtual ones. We denote by $h_{t}$ the $t$-th component of $h$.

In general we denote by the letter $\Pi$ probabilities of team wins. At time $t$ in history $h$ let $\Pi_{0}(h, t)$ be the probability that the team which shoots at $t$ wins, before the $t$-th kick has taken place. This number depends only the history up to $t-1$. Let $\Pi_{+}(h, t)$ and $\Pi_{-}(h, t)$ denote respectively the probabilities that the kicker's team wins knowing the history up to $t-1$ and knowing that the $t$-th kick has scored (index + ) or not (index -). By definition:

$$
\Pi_{0}(h, t)=p \Pi_{+}(h, t)+(1-p) \Pi_{-}(h, t) .
$$

We will now see how to compute these probabilities. It has to be done by backward induction, starting from the last dates.

Under the hypothesis that any kick scores with the same probability, it is clear that if the score is tie after the 10th shoot, the first team will eventually win with probability $1 / 2$. We can simply denote by $\Pi_{0}(h, 11)$ the outcome of the series of 10 shots, this number is therefore either 0,1 or $1 / 2$.

Then notice that, for any $t$, knowing the values of $\Pi_{0}(h, t+1)$ at $t+1$ for all $h$, one can find the values of $\Pi_{+}\left(h^{\prime}, t\right)$ and $\Pi_{-}\left(h^{\prime}, t\right)$ at $t$ :

- If $h_{t}=0$ then $\Pi_{-}(h, t)=1-\Pi_{0}(h, t+1)$ and $\Pi_{+}(h, t)=1-\Pi_{0}\left(h^{\prime}, t+1\right)$ where $h^{\prime}$ is any history that is identical to $h$ up to $t-1$ but $h_{t}^{\prime}=1$.

- Likewise if $h_{t}=1$ then $\Pi_{+}(h, t)=1-\Pi_{0}(h, t+1)$ and $\Pi_{-}(h, t)=$ $1-\Pi_{0}\left(h^{\prime}, t+1\right)$ where $h^{\prime}$ is any history that is identical to $h$ up to $t-1$ but $h_{t}^{\prime}=0$.

Then, for any $h$ and $t, \Pi_{0}(h, t)$ is deduced from $\Pi_{+}(h, t)$ and $\Pi_{-}(h, t)$ through equation (1), and one can see that this recursion defines all required numbers. 
For 10 kicks there are 1024 possible histories. We wrote a computer program to do these computations.

We are interested in two parameters. First, $\Pi_{0}$ captures the current state of affairs, somehow like the current score difference, but in a more discerning manner. Call $\Pi_{0}$ the achievement. Second, the difference $\Pi_{+}-\Pi_{-}$quantifies what is currently at stake and can be seen as the responsibility of the current player. Call $\Pi_{+}-\Pi_{-}$the stake.

Figure 1 displays the possible values of these numbers until the game is over. (For readability, we added a small random term so that more frequent values appear as darker squares.)
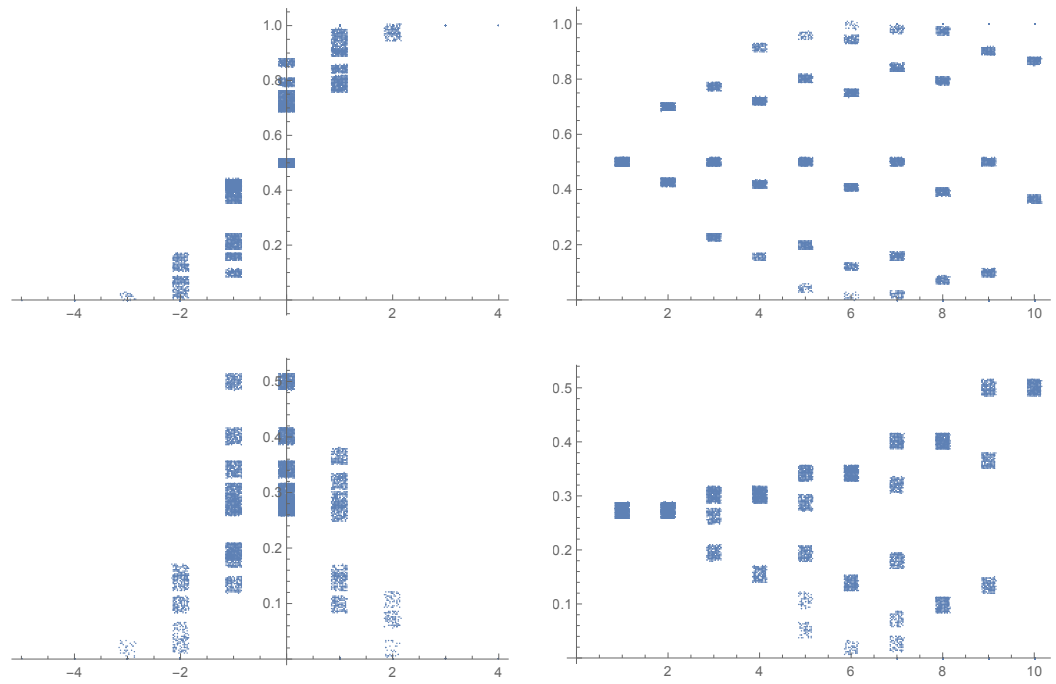

Figure 1 - Possible values of the achievement $\Pi_{0}$ (up) and the stake $\Pi_{+}-\Pi_{-}$ (down), by score difference (left) and by kick number (right).

As can be seen in the first line of Figure 1 the achievement is, obviously, much correlated with the score difference, but not equal to it. Its value is $1 / 2$ for the first shooter and it quickly takes a whole range of values.

The stake is the largest for a kick that will bring the probability the team to win from $1 / 2$ to 1 or from $1 / 2$ to 0 . This is always the case for the 10 th shooter, when the game is not already over. It may also be the case for the 9 th shooter. One can see in the picture that many different cases can occur, including high or low stakes in the middle of the series. 